\title{
Vasodilator and Constrictor Actions of Platelet-activating Factor in the Isolated Microperfused Afferent Arteriole of the Rabbit Kidney \\ Role of Endothelium-derived Relaxing Factor/Nitric Oxide and Cyclooxygenase Products
}

Luis A. Juncos, YiLin Ren, Shuji Arima, and Sadayoshi Ito

Hypertension and Vascular Research Division, Department of Medicine and Heart

and Vascular Institute, Henry Ford Hospital, Detroit, Michigan 48202.

\begin{abstract}
It has been suggested that platelet-activating factor (PAF) plays a prominent role in the control of glomerular hemodynamics in various physiological and pathological conditions. We examined the direct effect of PAF on rabbit glomerular afferent arterioles (Af-Arts) microperfused in vitro and tested whether endothelium-derived relaxing factor/nitric oxide (EDNO) and cyclooxygenase products are involved in its actions. In nanomolar concentrations PAF caused dose-dependent constriction of Af-Arts, with the maximum constriction being $34 \pm 10 \%$ at $4 \times 10^{-8} \mathrm{M}(n=9, P<0.001)$. The constriction was blunted by cyclooxygenase inhibition $(11 \pm 6 \%, n=7$, $P<0.05)$ but augmented by EDNO inhibition $(76 \pm 14 \%, n=8$, $P<0.005$ ). To study a possible vasodilator effect of PAF, Af-Arts were preconstricted with norepinephrine and increasing concentrations of PAF added to the lumen. At picomolar concentrations (lower than those that caused constriction), PAF produced dose-dependent vasodilation that was unaffected by cyclooxygenase inhibition but was abolished by EDNO synthesis inhibition. Both PAF-induced constriction and dilation of Af-Arts were blocked by a PAF receptor antagonist. This study demonstrates that PAF has a receptor-mediated biphasic effect on rabbit Af-Arts, dilating them at low concentrations while constricting them at higher concentrations. Our results suggest that PAF's vasodilator action may be due to production of EDNO, while its constrictor action is mediated at least in part through cyclooxygenase products. ( $J$. Clin. Invest. 1993. 91:1374-1379.) Key words: renal circulation • microvasculature • endothelium-derived relaxing factor • prostaglandins $\bullet$ thromboxane
\end{abstract}

\section{Introduction}

Platelet-activating factor(PAF) ${ }^{1}$, or 1-O-alkyl-2-acetyl-sn-glycero-3-phosphorylcholine, is a unique phospholipid with a broad range of biological activities. It is not only an important mediator in allergic and inflammatory reactions (1) but also a very potent hypotensive lipid with complex effects on the cardiovascular system $(1,2)$.

Address reprint requests to Dr. Sadayoshi Ito, Hypertension \& Vascular Research Division, Henry Ford Hospital, 2799 W. Grand Blvd., Detroit, MI 48202.

Received for publication 26 June 1992 and in revised form 22 October 1992.

1. Abbreviations used in this paper: EDNO, endothelium-derived relaxing factor/nitric oxide; PAF, platelet-activating factor; VSMC, vascular smooth muscle cells.

J. Clin. Invest.

(c) The American Society for Clinical Investigation, Inc. 0021-9738/93/04/1374/06 \$2.00

Volume 91, April 1993, 1374-1379
PAF is synthesized and released by many tissues, including the kidney (3-5). In fact, there is evidence suggesting that under normal conditions, the kidney is a major source of the PAF found in blood (6-9). Consequently, it has been speculated that PAF concentrations within the kidney may be sufficient to modulate renal hemodynamics. However, PAF's renal hemodynamic effects are poorly understood, and to our knowledge its direct actions on afferent arteriolar resistance have not been studied. Since the afferent arteriole accounts for most preglomerular resistance and plays a major role in the control of glomerular hemodynamics, it is important to understand the direct effects of PAF on this vascular segment.

PAF is known to dilate some vascular beds and constrict others $(1,10,11)$; however, the mechanisms by which it produces its effects are not known. It has been shown that PAF-induced dilation of the mesenteric arterial bed requires an intact endothelium (10), suggesting that endothelium-derived relaxing factor/nitric oxide (EDNO) may be responsible for PAFinduced dilation. Other studies also suggest that prostaglandin synthesis may be involved $(12,13)$. On the other hand, the vasoconstrictor actions of PAF may be due to a direct action on vascular smooth muscle cells (VSMC). This is supported by the findings that VSMC have PAF receptors (14) and that PAF increases intracellular calcium and activates protein kinase $C$ in cultured VSMC (15). However, PAF is also reported to stimulate production of thromboxane in the isolated perfused kidney $(13,16)$, and some studies have found that cyclooxygenase inhibitors attenuate PAF-induced vasoconstriction (17, 18). Consequently, it has been proposed that PAF induces renal vasoconstriction at least in part through the release of cyclooxygenase products such as thromboxane and prostaglandin endoperoxide.

In this study, we used the isolated microperfused rabbit afferent arteriole preparation to study the direct actions of PAF in the absence of confounding systemic hemodynamic and neurohumoral influences. We found that PAF has a receptormediated biphasic effect, dilating afferent arterioles at very low concentrations while constricting them at higher concentrations. We present evidence that PAF-induced dilation of afferent arterioles may be due to production of EDNO, while the vasoconstrictor response to higher concentrations of PAF may be due at least in part to metabolites of the cyclooxygenase pathway.

\section{Methods}

\section{Isolation and microperfusion of the afferent arteriole}

We used a method similar to that described previously to isolate and microperfuse afferent arterioles (19-21). Briefly, kidneys of young male New Zealand white rabbits (1.4-2.2 kg) were removed and sliced along the corticomedullary axis, and the slices were placed in ice-cold minimum essential medium (Gibco Laboratories, Grand Island, NY) 
containing 5\% BSA (Sigma Chemical Co., St. Louis, MO). From these slices, a single superficial afferent arteriole with its glomerulus intact was microdissected under a stereomicroscope ( $\mathrm{SZH}$; Olympus) as described previously (19-21). Using a micropipette, we transferred the arteriole to a temperature-regulated chamber mounted on the stage of an inverted microscope with Hoffman modulation (IMT-2, Olympus or Diaphot, Nikon ). The afferent arteriole was then cannulated with an array of glass pipettes as described previously $(22,23)$. Intraluminal pressure was measured by the Landis technique, using a fine pipette introduced into the arteriole through the perfusion pipette. The afferent arteriole was perfused with oxygenated medium 199 containing 5\% BSA, and intraluminal pressure was maintained at $60 \mathrm{mmHg}$ throughout the experiment.

The bath was identical to the arteriolar perfusate (except that it contained $0.1 \%$ BSA) and was exchanged continuously. Microdissection and cannulation of the afferent arteriole were completed within 90 min at $8^{\circ} \mathrm{C}$, after which the bath was gradually warmed to $37^{\circ} \mathrm{C}$ for the rest of the experiment. Once the temperature was stable, a 30 -min equilibration period was allowed before taking any measurements. Images of the afferent arteriole were displayed at magnifications up to 1,980 and recorded with a video system consisting of a camera (DXC755; Sony, Tokyo), monitor (MA PVM-1942Q; Sony), and video recorder (EDV-9500; Sony). The diameter was measured with an image analysis system (Fryer, Carpentersville, IL).

In the experiments that examined PAF's vasoconstrictor actions (protocols 1, 3a, 6, and 7), PAF was added to both the bath and lumen, because in preliminary studies the vasoconstrictor responses tended to be greater when PAF was added in this manner. On the other hand, PAF-induced vasodilation in other vascular beds has been suggested to be due to PAF's effect on the endothelium (10); therefore, we added PAF only to the lumen when studying its vasodilator effects (protocols $2,3 b, 4$, and 5 ).

\section{Experimental protocols}

Response of afferent arterioles to $P A F$. After the 30-min equilibration period, increasing doses of PAF $\left(4 \times 10^{-11}\right.$ to $4 \times 10^{-8} \mathrm{M}$; Sigma $)$ were added to both bath and lumen. Luminal diameter was measured immediately before adding PAF and observed for at least $10 \mathrm{~min}$ at each dose.

Response of preconstricted afferent arterioles to $P A F$. To determine whether PAF dilates afferent arterioles, they were preconstricted to approximately $50 \%$ of basal diameter by adding norepinephrine (Sigma) to the bath, after which increasing doses of PAF $\left(4 \times 10^{-13}\right.$ to 4 $\times 10^{-8} \mathrm{M}$ ) were added to the lumen.

Effect of an antagonist of PAF on its vascular actions. To test whether the effects of PAF were specific and receptor-mediated, we used $10^{-4} \mathrm{M}$ hexanolamino-PAF C-16 (Cayman Chemical Corp., Ann Arbor, MI), a PAF antagonist $(24,25)$. We first tested whether the antagonist altered PAF-induced constriction. For this, we added the antagonist to the bath and arteriolar perfusate immediately following the equilibration period. After allowing a 15 -min period, the effect of PAF was examined as described in protocol 1.

We next tested whether the PAF antagonist could block the vasodilator action of PAF. We preconstricted the afferent arteriole with norepinephrine as in protocol 2 and then added the antagonist to the bath and arteriolar perfusate. 15 min later, increasing concentrations of PAF $\left(4 \times 10^{-13}\right.$ to $\left.4 \times 10^{-11} \mathrm{M}\right)$ were added to the lumen.

Effect of cyclooxygenase inhibition on PAF-induced vasodilation. Since PAF is known to stimulate production of prostaglandins, we tested whether a cyclooxygenase inhibitor affects PAF-induced vasodilation. Indomethacin (Sigma) was added to the bath and arteriolar perfusate at a final concentration of $5 \times 10^{-5} \mathrm{M}$ from the equilibration period to the end of the experiment. We preconstricted the afferent arteriole with norepinephrine as described in protocol 2 and examined the effect of intraluminal PAF $\left(4 \times 10^{-13}\right.$ to $\left.4 \times 10^{-11} \mathrm{M}\right)$.

Effect of EDNO synthesis inhibition on $P A F$-induced vasodilation. To determine whether EDNO mediates PAF-induced vasodilation, we added $10^{-4} \mathrm{M} \mathrm{N}^{\mathrm{w}}$-nitro-L-arginine (L-NAME; Sigma), which inhibits synthesis of EDNO (26), to the arteriolar perfusate after the equilibra- tion period. $15 \mathrm{~min}$ later, the arteriole was preconstricted with norepinephrine and the effect of PAF examined as in protocol 3. We have previously reported that this concentration of L-NAME abolishes acetylcholine-induced vasodilation in this preparation (23).

Effect of EDNO synthesis inhibition on PAF-induced constriction. We found that L-NAME blocked PAF-induced vasodilation (see Results), suggesting that PAF enhances EDNO synthesis. The enhanced EDNO synthesis might in turn be an important mechanism counteracting PAF-induced vasoconstriction. If so, augmentation of PAF-induced vasoconstriction should be apparent when EDNO synthesis is inhibited. To test this possibility, afferent arterioles were pretreated with L-NAME $\left(10^{-4} \mathrm{M}\right)$ as described in protocol 4 , after which increasing doses of PAF $\left(4 \times 10^{-11}\right.$ to $\left.4 \times 10^{-8} \mathrm{M}\right)$ were added to the bath and lumen.

Effect of cyclooxygenase inhibition on PAF-induced vasoconstriction. We examined whether metabolites of the cyclooxygenase pathway (such as thromboxane $A_{2}$ and prostaglandin endoperoxide) are involved in PAF-induced constriction. The experimental design was identical to protocol 1 except that afferent arterioles were pretreated with indomethacin $\left(5 \times 10^{-5} \mathrm{M}\right)$.

\section{Statistics}

Values were expressed as mean \pm SEM. Univariate repeated-measures ANOVA with the Greenhouse-Geisser sphericity correction was used to test for an overall change from the control to the $4 \times 10^{-8} \mathrm{M}$ period (constriction study) or from the norepinephrine to the $4 \times 10^{-11} \mathrm{M}$ period (dilation study). Given the presence of an overall change across periods at the 0.05 significance level, paired $t$ tests were used to examine whether the diameter at a given dose differed from the baseline (constriction data) or from the norepinephrine value (dilation data). Bonferroni's multiple comparison adjustment was used to reduce the significance level of each paired $t$ test from 0.05 to 0.013 in the constriction study and from 0.05 to 0.017 in the dilation study.

Univariate repeated-measures ANOVA with the GreenhouseGeisser sphericity correction was used to test whether the groups differed with respect to the rate of change across the various periods. For this analysis, each experimental period was represented in terms of its percent change from the control period. Given the presence of a significant or borderline interaction effect, Student's two-sample $t$ test was used to test for a group difference at each experimental period. Bonferroni's multiple comparison adjustment was used to reduce the significance level of each paired $t$ test from 0.05 to 0.013 .

\section{Results}

Response of afferent arterioles to PAF. Basal luminal diameter was $16.5 \pm 0.9 \mu \mathrm{m}$ and was not affected by vehicle $(n=8)$. On the other hand, PAF caused a dose-dependent decrease in luminal diameter $(P<0.005 ; n=9)$ (Fig. 1). Luminal diameter decreased from a basal diameter of $15.7 \pm 1.4 \mu \mathrm{m}$ to $13.7 \pm 1.3$ ( $P<0.005$ vs. basal diameter $), 12.5 \pm 1.4(P<0.005)$, and $10.1 \pm 1.2 \mu \mathrm{m}(P<0.005)$ at $4 \times 10^{-10}, 4 \times 10^{-9}$, and $4 \times 10^{-8}$ $M$, respectively; this represented a decrease of $12.9 \pm 2.3$, $20.3 \pm 4.2$, and $34.1 \pm 7.8 \%$. Full response to PAF was attained within $5 \mathrm{~min}$ after application and persisted throughout the 10-min period.

Response of preconstricted afferent arterioles to PAF. Norepinephrine decreased the luminal diameter to $50.6 \pm 4.8 \%$ of basal diameter, from $16.6 \pm 0.5$ to $8.2 \pm 0.8 \mu \mathrm{m}$ (Fig. 2 ). Addition of vehicle to the lumen had no effect $(n=8)$. In contrast, when PAF was added to arterioles preconstricted to $40.5 \pm 4.5 \%$ of basal diameter (from $16.2 \pm 0.9$ to $6.5 \pm 0.6 \mu \mathrm{m}$ ), diameter increased in a dose-dependent manner at low concentrations $(P<0.005 ; n=7)$. Maximal dilation was obtained at $4 \times 10^{-11}$ $\mathrm{M}$, which increased the diameter to $12.7 \pm 0.6 \mu \mathrm{m}(80.4 \pm 6.8 \%$ of basal values; $P<0.005$ ); however, when PAF concentra- 


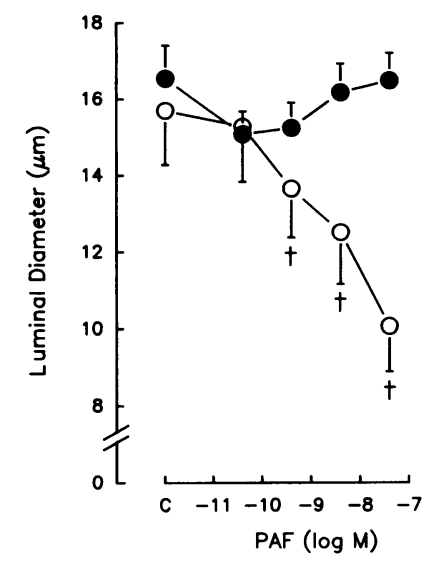

Figure 1. Effect of PAF $(0)(n=$ 9) or vehicle $(\bullet)(n=8)$ on the luminal diameter of the afferent arteriole. ${ }^{\dagger} P<0.013 v s$. basal diameter.

tions were increased further, luminal diameter decreased, reaching $8.0 \pm 0.5 \mu \mathrm{m}$ at $4 \times 10^{-8} \mathrm{M}$.

Effect of an antagonist of PAF on its vascular actions. Addition of the PAF antagonist did not affect luminal diameter (15.2 \pm 1.2 vs. $14.6 \pm 1.1 \mu \mathrm{m} ; n=4$ ) but blocked PAF's vasoconstrictor action $(14.9 \pm 1.3,14.8 \pm 1.5$ and $14.5 \pm 1.3 \mu \mathrm{m}$ at 4 $\times 10^{-10}, 4 \times 10^{-9}$ and $4 \times 10^{-8} \mathrm{M}$ PAF, respectively). Likewise, in arterioles preconstricted with norepinephrine from $16.4 \pm 1.0$ to $9.5 \pm 0.8 \mu \mathrm{m}$, the PAF antagonist by itself did not alter luminal diameter ( $8.8 \pm 0.8 \mu \mathrm{m} ; n=4)$ but abolished PAFinduced dilation $(8.9 \pm 1.1,8.5 \pm 1.3$, and $9.0 \pm 1.4 \mu \mathrm{m}$ at 4 $\times 10^{-13}, 4 \times 10^{-12}$, and $4 \times 10^{-11}$ M PAF, respectively).

Effect of cyclooxygenase inhibition on PAF-induced vasodilation. Pretreatment with indomethacin did not alter basal diameter $(14.4 \pm 0.9 \mu \mathrm{m} ; n=6)$, nor did it alter the vasodilator response of preconstricted afferent arterioles to PAF. When PAF was added to arterioles preconstricted to $6.6 \pm 0.4 \mu \mathrm{m}$ (47.2 $\pm 3.4 \%$ of basal diameter), diameter increased to the same extent as in the nontreated arterioles; the diameter was $8.6 \pm 0.8,11.1 \pm 1.0$, and $13.4 \pm 0.6 \mu \mathrm{m}$ at $4 \times 10^{-13}, 4 \times 10^{-12}$, and $4 \times 10^{-11} \mathrm{M}$, respectively.

Effect of EDNO synthesis inhibition on PAF-induced vasodilation. After pretreatment, basal luminal diameter decreased by $17.9 \pm 3.2 \%$, falling from $15.1 \pm 0.9$ to $12.4 \pm 0.6 \mu \mathrm{m}(n=7 ; P$ $<0.05)$. Fig. 3 shows an example of the arteriolar response to $40 \mathrm{pM}$ PAF in nontreated (left) and L-NAME-treated Af-Arts (right), both of which were preconstricted with norepinephrine. As clearly seen, L-NAME abolished PAF-induced vasodilation (ANOVA interaction, $P<0.001$ ). Fig. 4 summarizes seven such experiments, with the results in nontreated arterioles from protocol 2 shown for comparison. After L-NAME pretreatment, arterioles were preconstricted further with norepinephrine to $46.6 \pm 3.6 \%$ of basal diameter (from $12.4 \pm 0.6$ to

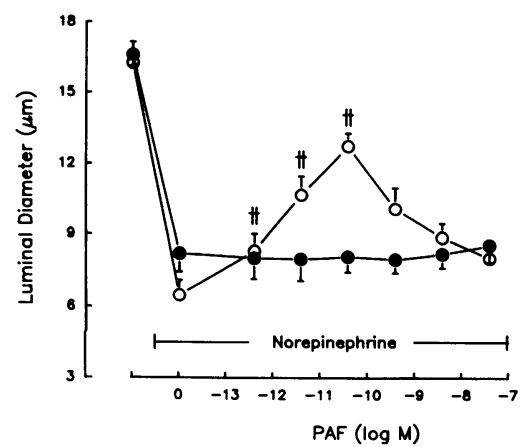

Figure 2. Effect of PAF (०) $(n=7)$ or vehicle (•) $(n=8)$ on the luminal diameter of afferent arterioles preconstricted with norepinephrine. " $P<0.017$ $v s$. pre-PAF values.

$5.7 \pm 0.4 \mu \mathrm{m})$. In these arterioles, PAF did not produce dilation, but rather tended to decrease the diameter further to $4.5 \pm 0.9$ and $3.7 \pm 1.0 \mu \mathrm{m}$ at $4 \times 10^{-12}$ and $4 \times 10^{-11} \mathrm{M}$, respectively.

Effect of EDNO synthesis inhibition on PAF-induced vasoconstriction. Fig. 5 shows an example of vasoconstrictor response to $4 \times 10^{-8}$ M PAF in L-NAME-pretreated and nontreated afferent arterioles. After L-NAME pretreatment, basal diameter decreased from $14.8 \pm 1.4$ to $11.5 \pm 1.7 \mu \mathrm{m}$ on average and PAF at $4 \times 10^{-11}, 4 \times 10^{-10}, 4 \times 10^{-9}$ and $4 \times 10^{-8} \mathrm{M}$ decreased the diameter further by $2.6 \pm 0.7,3.7 \pm 0.5,5.6 \pm 1.2$, and $8.6 \pm 1.2 \mu \mathrm{m}$, respectively. These values represent a $22.2 \pm 4.6,35.17 \pm 4.7,51.8 \pm 9.2$, and $75.8 \pm 5.6 \%$ decrease in the luminal diameter, respectively $(n=8)$, which were significantly larger than in the nontreated arterioles (Fig. 6).

Effect of cyclooxygenase inhibition on PAF-induced vasoconstriction. Pretreatment with indomethacin did not alter the basal diameter ( $13.9 \pm 1.4 \mu \mathrm{m} ; n=7)$; however, it attenuated PAF-induced constriction. Indeed, indomethacin pretreatment completely blocked the vasoconstriction induced by PAF at $4 \times 10^{-10}$ and $4 \times 10^{-9} \mathrm{M}$. Only at $4 \times 10^{-8} \mathrm{M}$ did PAF tend to cause constriction ( $\Delta=1.8 \pm 0.8 \mu \mathrm{m}$, representing an $11.6 \pm 5.2 \%$ decrease $)$.

\section{Discussion}

In the present study, we investigated the direct effects of PAF on the afferent arteriole as well as the possible pathways through which PAF makes its effects felt. We found that in the isolated microperfused rabbit afferent arteriole, PAF has a biphasic effect, inducing vasodilation at very low concentrations and vasoconstriction at higher concentrations. Both of these effects appear to be receptor mediated, since they were blocked by a specific PAF receptor antagonist. Furthermore, our results suggest that PAF-induced vasodilation may be due to production of EDNO, whereas PAF-induced vasoconstriction may be due at least in part to metabolites of the cyclooxygenase pathway.

Several studies have shown that PAF produces renal vasodilation in vivo $(27,28)$ and in isolated perfused rat kidneys (29). In contrast, other studies $(17,30,31)$ have reported that intrarenal infusion of PAF decreases renal blood flow and glomerular filtration rate in rats. The reason for this discrepancy is not clear; it may be related to the dose employed, or, alternatively, systemic hemodynamic and/or neurohormonal changes secondary to PAF infusion may have influenced the results. In the present study we examined the direct action of PAF on isolated microperfused afferent arterioles. We observed vasodilation in preconstricted arterioles at very low concentrations, while concentrations of $4 \times 10^{-10} \mathrm{M}$ and higher induced constriction. Thus the contradictory findings of previous studies might have been due to differences in dosage. Indeed, in the studies by Badr et al. (17), Wang and Dunn (30) and Tolins et al. (31), estimated that concentrations of PAF in the renal circulation were between $5 \times 10^{-10}$ and $2 \times 10^{-9} \mathrm{M}$, whereas Handa et al. (27), who observed vasodilation, estimated that concentrations were 25 - to 100 -fold lower. Therefore, these studies are consistent with our findings.

In the present study, the diameter of preconstricted afferent arterioles increased in a dose-dependent manner until PAF reached $4 \times 10^{-11} \mathrm{M}$ and then began to decrease. The decrease in diameter was similar to that observed in non-preconstricted arterioles, suggesting that it was due to the vasoconstrictor action of PAF. However, since desensitization to PAF has been 

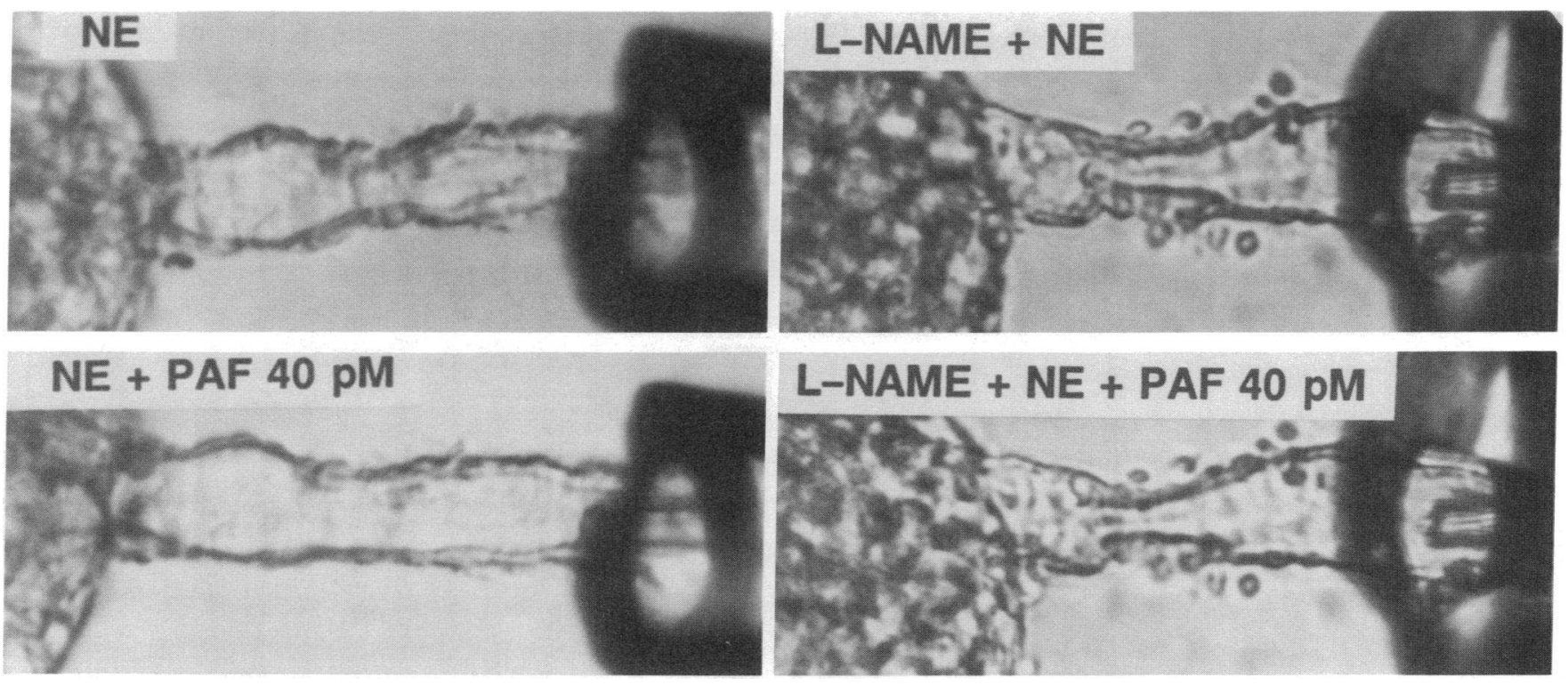

Figure 3. Example of arteriolar response to 40 pM PAF in nontreated and L-NAME-pretreated afferent arterioles preconstricted with norepinephrine. Note that PAF induced strong vasodilation, which was abolished by L-NAME.

reported in a variety of cell types (15), it could be argued that the decrease in diameter was due to waning of the vasodilator action of PAF rather than vasoconstriction. We addressed this possibility by decreasing the concentration of PAF from 4 $\times 10^{-8}$ to $4 \times 10^{-11} \mathrm{M}$, which caused the luminal diameter to increase once again (data not shown). Therefore, it is unlikely that the decrease in diameter observed at higher concentrations is due to waning of PAF-induced vasodilation.

While PAF-induced renal vasodilation has been reported previously, the mechanism by which it dilates the renal vasculature is unknown. PAF has been found to stimulate the release of vasodilator prostaglandins in a variety of vascular beds ( 1 , $12)$ including the kidney $(1,4,13)$, raising the possibility that they may be involved in PAF-induced renal vasodilation. However, both Handa et al. (27) and Schwertschlag et al. (29) reported that blocking prostaglandin synthesis with cyclooxygenase inhibitors failed to inhibit PAF-induced renal vasodilation. In this study we also found that blocking cyclooxygenase with indomethacin failed to alter PAF-induced vasodilation of afferent arterioles. Consequently, these studies provide evidence that prostaglandins may play no role in the renal vasodilator actions of PAF.

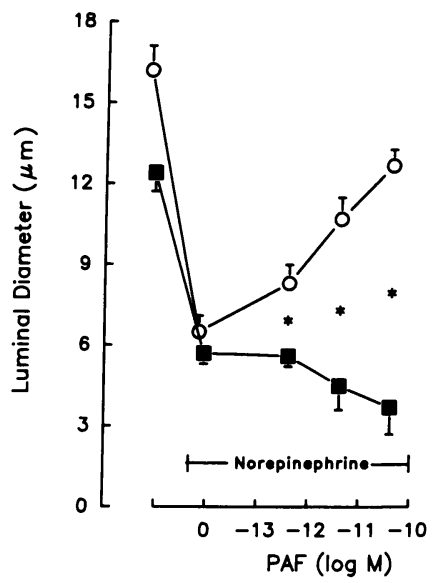

Figure 4. Effect of PAF on luminal diameter in L-NAMEtreated $(\square)(n=7)$ and nontreated $(0)(n=7)$ afferent arterioles. Note that L-NAMEpretreated afferent arterioles did not dilate in response to PAF $\left({ }^{*} P<0.013\right.$ vs. nontreated arterioles) but rather tended to constrict further.
We $(23,32)$ and others (33) have demonstrated that acetylcholine dilates isolated afferent arterioles preconstricted with endothelin or norepinephrine, suggesting that the renal microvasculature can produce EDNO. Furthermore, this locally produced EDNO may be an important modulator of the vascular response to angiotensin II and endothelin $(23,32)$. However, the contribution of EDNO to PAF's actions in the kidney is poorly understood. In this study, pretreatment with L-NAME abolished PAF-induced dilation of preconstricted afferent arterioles, suggesting that PAF-induced dilation depends on EDNO synthesis. When EDNO is inhibited by L-NAME, 4 $\times 10^{-12}$ and $10^{-11} \mathrm{M}, \mathrm{PAF}$ - which otherwise caused dilation -now produced further constriction. Moreover, pretreatment with L-NAME potentiated the vasoconstrictor response to PAF in non-preconstricted arterioles. These observations suggest that EDNO synthesis is not only critical in mediating PAF's vasodilator effects, but also an important modulator of PAF-induced vasoconstriction. Thus, under conditions of suppressed EDNO activity, PAF is predominantly a vasoconstrictor even at low concentrations. Consistent with the hypothesis that PAF stimulates EDNO synthesis, PAF has recently been shown to cause an increase in intracellular calcium in cultured endothelial cells $(34,35)$, which is known to stimulate EDNO synthesis. In addition, a recent study (36) reported that PAF stimulates release of EDNO in cultured endothelial cells derived from the bovine renal glomerulus.

Several studies have suggested that the vasoconstrictor effects of PAF depend on generation of eicosanoid mediators such as thromboxanes, prostaglandin endoperoxide and leukotrienes. For instance, Badr et al. (17) and Plante et al. (37) found that pretreatment with either indomethacin or a prostaglandin endoperoxide/thromboxane receptor antagonist blocks the reduction of glomerular filtration rate and renal blood flow induced by PAF. However, other studies $(4,38)$ have shown that indomethacin either augments PAF-induced renal vasoconstriction or has no effect. We found that indomethacin pretreatment significantly blunted PAF-induced vasoconstriction. Only at $4 \times 10^{-8} \mathrm{M}$ did PAF tend to constrict indomethacin-treated arterioles; however, this constriction was 

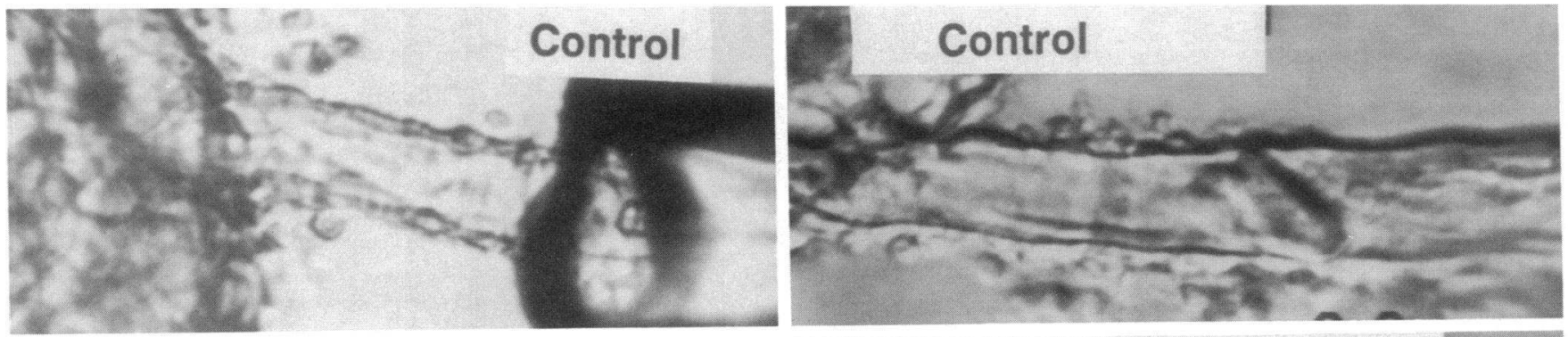

\section{$50 \mu \mathrm{m}$}
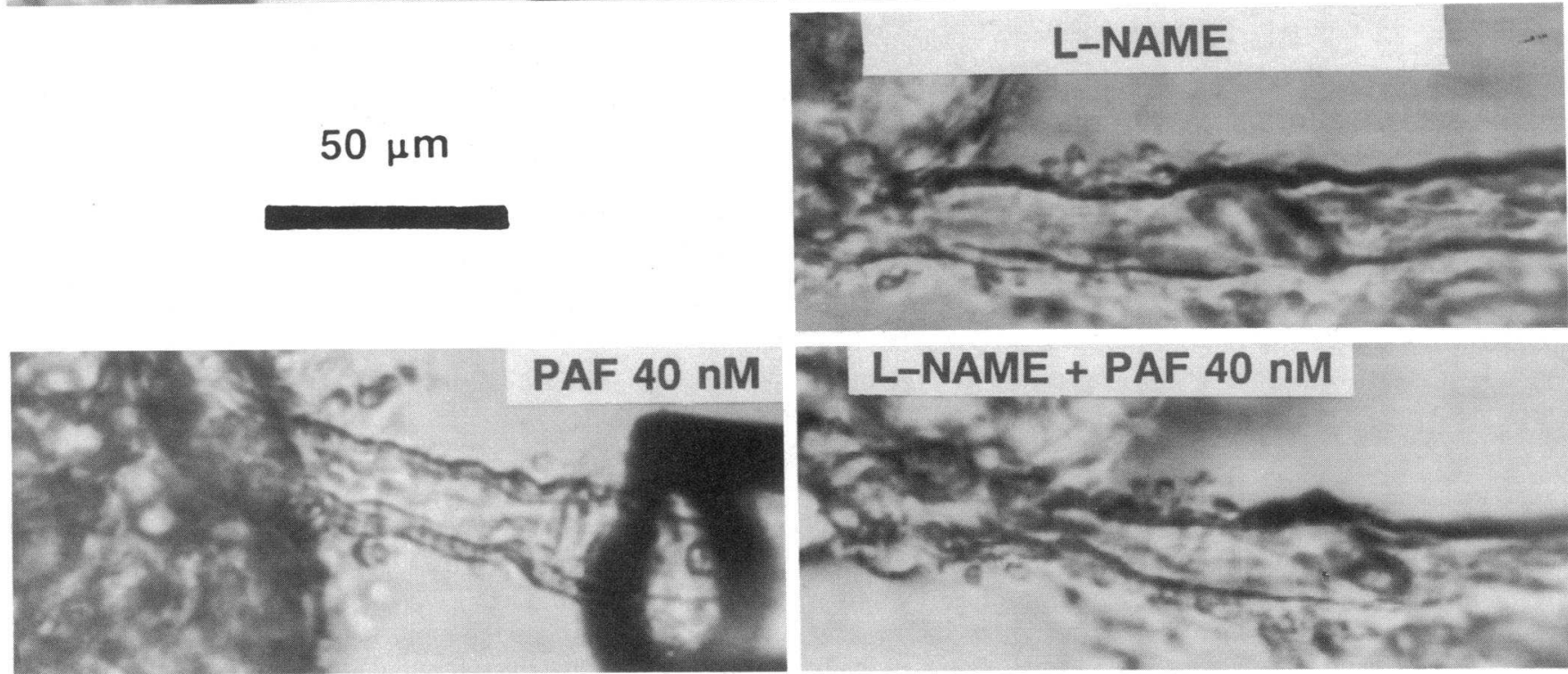

Figure 5. Example of arteriolar response to $40 \mathrm{nM}$ PAF in nontreated and L-NAME-pretreated afferent arterioles. Note that L-NAME reduced the basal diameter and augmented the vasoconstrictor response to PAF.

much weaker than that seen in nontreated arterioles. While our results suggest a role for cyclooxygenase metabolites (e.g., $\mathrm{PGH}_{2} / \mathrm{TxA}_{2}$ ) in PAF-induced vasoconstriction, we cannot exclude the possibility that blockade of cyclooxygenase may cause arachidonic acid to be shunted into other pathways (e.g., epoxygenase or lipoxygenase pathways), which could have influenced the results.

PAF is known to bind to specific receptors and stimulate phospholipase $\mathrm{C}$ activity, with concomitant increases in intracellular levels of phosphoinositides, calcium and diacyl-

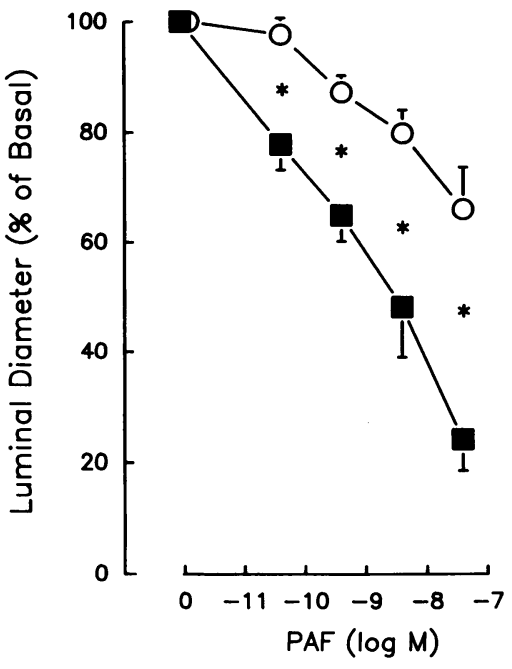

Figure 6. Effect of PAF on luminal diameter in L-NAME-treated $(\bullet)(n$ $=8)$ and nontreated (O) $(n=9)$ afferent arterioles. Note that LNAME pretreatment augmented the vasoconstrictor response to $\operatorname{PAF}\left({ }^{*} P<0.013\right.$ vs. nontreated arterioles). glycerol, in both endothelial cells and $\operatorname{VSMC}(14,15,34,35)$. Therefore, the net effect of PAF may depend upon the cell type on which it is predominantly acting. Our study suggests that PAF's vasodilator action is likely due to an effect on the endothelial cells because of its rapid onset of action and reversibility. While we cannot exclude the possibility that PAF may induce nitric oxide synthase in VSMC, it is unlikely that this would account for the vasodilation observed in our study, since induction of VSMC nitric oxide synthase requires hours and is not easily reversed (39). On the other hand, PAF-induced vasoconstriction could be due to stimulation of $\mathrm{PGH}_{2} / \mathrm{TxA}_{2}$ activity in endothelial cells as well as its direct effect on VSMC.

Our study demonstrates the importance of PAF concentration and endothelial status in determining its effect in the afferent arteriole. Although the concentration of PAF normally found in the glomerular microenvironment is not known, there is evidence that renal PAF levels reach biologically active concentrations in a number of experimental and clinical conditions $(1-5,7,8,16,30,40-43)$. For instance, after the kidney is unclipped in either two-kidney-one-clip or one-kidney-oneclip Goldblatt hypertension, renal medullary interstitial cells release a large amount of PAF into the renal venous effluent $(2$, 5,8 ). This is accompanied by a decrease in peripheral resistance and a fall in blood pressure, both of which are markedly attenuated by infusion of a PAF antagonist $(2,5,43)$. On the other hand, PAF may be released directly into the glomerular microenvironment by mesangial and endothelial cells, as well as by activated leukocytes and platelets $(3,4,16,40,42)$ in certain pathological conditions. Indeed, in experimental 
nephrotoxic nephritis, PAF concentrations are reportedly increased (4, 16, 40-42), while PAF antagonists substantially ameliorate the reduction in glomerular filtration rate and renal plasma flow as well as in proteinuria and histological lesions (40-42). This suggests that increased PAF may cause contraction of the renal microvasculature and/or glomerular mesangial cells and also alter glomerular permselectivity. Although endothelial function was not known in these studies, there are clinical situations where increased renal levels of PAF coexist with morphological evidence of endothelial damage, such as lupus nephritis and acute allograft rejection, in which case PAF's constrictor actions may be even stronger.

In conclusion, we have shown that even at picomolar concentrations PAF causes strong vasodilation in the rabbit afferent arteriole, which is highly dependent upon intact EDNO synthesis. However, when EDNO synthesis is impaired, PAF's vasoconstrictor action becomes apparent at very low concentrations and stronger at higher concentrations. Thus the functional integrity of the endothelium may be a critical factor that determines the glomerular hemodynamic actions of PAF in various pathophysiological conditions.

\section{Acknowledgments}

This study was supported by National Institutes of Health grants HL46518 and HL-28982.

\section{References}

1. Braquet, P., L. Touqui, T. Y. Shen, and B. B. Vargaftig. 1987. Perspectives in platelet-activating factor research. Pharmacol. Rev. 39:97-145.

2. Vandongen, R. 1991. Platelet-activating factor and the circulation. J. Hypertens. 9:771-778.

3. Schlondorff, D., P. Goldwasser, R. Neuwirth, J. Satriano, and K. Clay. 1986. Production of platelet-activating factor in glomeruli and cultured glomerular mesangial cells. Am. J. Physiol. 250:F1123-F1127.

4. Schlondorff, D., and R. Neuwirth. 1986. Platelet-activating factor and the kidney. Am. J. Physiol. 251:F1-F11.

5. Muirhead, E. 1986. Renomedullary system of blood pressure control. $\mathrm{Hy}$ pertens. 8(Suppl I):I-38-I-46.

6. Caramelo, C., S. Fernandes-Gallardo, D. Marin-Cao, P. Inarrea, J. C. Santos, J. Lopez-Novoa, and M. Sanchez-Crespo. 1984. Presence of platelet-activating factor in blood from humans and experimental animals. Its absence in anephric individuals. Biochem. Biophys. Res. Commun. 120:789-796.

7. Lee, T., B. Malone, D. Woodward, and F. Snyder. 1989. Renal necrosis and the involvement of a single enzyme of the de novo pathway for the biosynthesis of platelet-activating factor in rat kidney inner medulla. Biochem. Biophys. Res. Commun. 163:1002-1005.

8. McGowan, H. M., R. Vandongen, J. P. Codde, and K. D. Croft. 1986. Increased aortic $\mathrm{PGI}^{2}$ and plasma lyso-PAF in the unclipped one-kidney hypertensive rat. Am. J. Physiol. 251:H1361-H1364.

9. Snyder, F. 1990. Platelet-activating factor and related acetylated lipids as potent biologically active cellular mediators. Am. J. Physiol. 259:C697-C708.

10. Kamata, K., T. Mori, K. Shigenobu, and Y. Kasuya. 1989. Endotheliumdependent vasodilator effects of platelet-activating factor on rat resistance vessels. Br. J. Pharmacol. 98:1360-1364.

11. Dillon, P. K., A. B. Ritter, and W. N. Duran. 1988. Vasoconstrictor effects of platelet-activating factor in the hamster cheek pouch microcirculation: doserelated relations and pathways of action. Circ. Res. 62:722-731.

12. Alving, K., R. Matran, and J. M. Lundberg. 1991. The possible role of prostaglandin $\mathrm{D}^{2}$ in the long lasting airways vasodilation induced by allergen in the sensitized pig. Acta Physiol. Scand. 143:93-103.

13. Weisman, S. M., D. Felsen, and E. D. Vaughan, Jr. 1990. Platelet-activating factor is a potent stimulus for renal prostaglandin synthesis: possible significance in unilateral ureteral obstruction. J. Pharmacol. Exp. Ther. 253:10.

14. Hwang, S., C. C. Lee, M. J. Cheah, and T. Y. Shen. 1983. Specific receptor sites for 1-0-alkyl-2-0-acetyl-sn-glycero-3-phosphocholine (platelet-activating factor) on rabbit platelet and guinea pig smooth muscle membranes. Biochemistry 22:4756-4763.

15. Schwertschlag, U. S., and A. R. Whorton. 1988. Platelet-activating factorinduced homologous and heterologous desensitization in cultured vascular smooth muscle cells. J. Biol. Chem. 263:13791-13796.

16. Camussi, G. 1986. Potential role of platelet-activating factor in renal pathophysiology. Kidney Int. 29:469-477.
17. Badr, K. F., D. K. DeBoer, K. Takahashi, R. C. Harris, A. Fogo, and H. R. Jacobson. 1989. Glomerular responses to platelet-activating factor in the rat: role of thromboxane $\mathrm{A}_{2}$. Am. J. Physiol. 256:F35-F43.

18. Yoo, J., D. Schlondorff, and J. Neugarten. 1990. Thromboxane mediates the renal hemodynamic effects of platelet-activating factor. J. Pharmacol. Exp. Ther. 253:743-748.

19. Itoh, S., O. A. Carretero, and R. D. Murray. 1985. Renin release from isolated afferent arterioles. Kidney Int. 27:762-767.

20. Itoh, S., and O. A. Carretero. 1985. Role of the macula densa in renin release. Hypertension. Suppl. I 7:I-49-I-54.

21. Ito, S., and O. A. Carretero. 1990. An in vitro approach to the study of macula densa-mediated glomerular hemodynamics. Kidney Int. 38:1206-1210.

22. Osgood, R. W., M. Patton, M. J. Hanley, M. Venkatachalam, H. J. Reineck, and J. H. Stein. 1983. In vitro perfusion of the isolated dog glomerulus. Am. J. Physiol. 244:F349-F354.

23. Ito, S., C. S. Johnson, and O. A. Carretero. 1991. Modulation of angiotensin II-induced vasoconstriction by endothelium-derived relaxing factor in the isolated microperfused rabbit afferent arteriole. J. Clin. Invest. 87:1656-1663.

24. Rouis, M., F. Nigon, and M. J. Chapman. 1988. Platelet activating factor is a potent stimulant of the production of active oxygen species by human monocyte-derived macrophages. Biochem. Biophys. Res. Commun. 156:1293-1301.

25. Tokumura, A., H. Homma, and D. Hanahan. 1985. Structural analogs of alkylacetylglycerophosphocholine inhibitory behavior on platelet activation. $J$. Biol. Chem. 260:12710-12714.

26. Moore, P. K., O. A. al-Swayeh, N. W. Chong, R. A. Evans, and A. Gibson. 1990. L-N ${ }^{G}$-nitro arginine ( L-NOARG), a novel, L-arginine-reversible inhibitor of endothelium-dependent vasodilation in vitro. Br. J. Pharmacol. 99:408-410.

27. Handa, R. K., J. W. Strandhoy, and V. M. Bucklew, Jr. 1990. Platelet-activating factor is a renal vasodilator in the anesthetized rat. Am. J. Physiol. 258:F1504-F1509.

28. Handa, R. K., J. W. Strandhoy, and V. M. Bucklew, Jr. 1991. Vasorelaxant effect of C16-PAF and C18-PAF on renal blood flow and systemic blood pressure in the anesthetized rat. Life Sci. 49:747-752.

29. Schwertschlag, U., H. Scherf, J. G. Gerber, M. Mathias, and A. S. Nies. 1987. Platelet activating factor induces changes on renal vascular resistance, vascular reactivity, and renin release in the isolated perfused rat kidney. Circ. Res. 60:534-539.

30. Wang, J., and M. Dunn. 1987. Platelet-activating factor mediates endotoxin-induced acute renal insufficiency in rats. Am. J. Physiol. 253:F1283F1289.

31. Tolins, J. P., G. M. Vercellotti, M. Wilkowske, B. Ha, H. S. Jacob, and L. Raij. 1989. Role of platelet activating factor in endotoxemic acute renal failure in the male rat. J. Lab. Clin. Med. 113:316-324.

32. Ito, S., L. A. Juncos, N. Nushiro, C. S. Johnson, and O. A. Carretero. 1991. Endothelium-derived relaxing factor modulates endothelin action in afferent arterioles. Hypertension 17:1052-1056.

33. Edwards, R. M. 1985. Response of isolated renal arterioles to acetylcholine, dopamine, and bradykinin. Am. J. Physiol. 248:F183-F189.

34. Bussolino, F., M. Aglietta, F. Sanavio, A. Stacchini, D. Lauri, and G. Camussi. 1985. Alkyl-ether phosphoglycerides influence calcium fluxes into human endothelial cells. J. Immunol. 135:2748-2753.

35. Camussi, G. 1986. Role of platelet-activating factor released from endothelial cells in the pathophysiology of the inflammatory reaction. In Proceedings of the First Sandoz Research Symposium. New Horizons in Platelet-Activating Factor Research. C. M. Winslow and M. L. Lee, editors. John Wiley \& Sons, Inc., London.

36. Marsden, P. A., T. S. Brock, and B. J. Ballermann. 1990. Glomerular endothelial cells respond to calcium mobilizing agonists with release of EDRF. Am. J. Physiol. 258:F1295-F1303.

37. Plante, G. E., R. L. Hebert, and P. Sirois. 1987. Inhibition of PAF-induced renal vasoconstriction by 1-655,240, a thromboxane/prostaglandin endoperoxide antagonist. Proc. Int. Symposium on Renal Eicosanoids. 21 A (Abstr.)

38. Scherf, H., A. S. Nies, U. Schwertschlag, M. Hughes, and J. G. Gerber. 1986. Hemodynamic effects of platelet activating factor in the dog kidney in vivo. Hypertension 8:737-741.

39. Moncada, S., R. M. Palmer, and E. A. Higgs. 1991. Nitric oxide: physiology, pathophysiology, and pharmacology. Pharmacol. Rev. 43:109-142.

40. Bertani, T., M. Livion, D. Macconi, M. Morigi, G. Bisogno, C. Patrono, and G. Remuzzi. 1987. Platelet activating factor (PAF) as a mediator of injury in nephrotoxic nephritis. Kidney Int. 31:1248-1256.

41. Felsen, D., M. Loo, D. Marion, and E. Vaughan, Jr. 1990. Involvement of platelet-activating factor and thromboxane $\mathrm{A}_{2}$ in the renal response to unilateral ureteral obstruction. J. Urol. 144:141-145.

42. Remuzzi, G. 1989. Eicosanoids and platelet-activating factor as possible mediators of injury in experimental nephropathies. Adv. Exp. Med. Biol. 259:221-247.

43. Masugi, F., T. Ogihara, A. Otsuka, S. Saeki, and Y. Kumahara. 1984. Effect of 1-alkyl-2-acetyl-sn-glycero-3-phosphorylcholine inhibitor on the reduction of one-kidney, one clip hypertension after unclipping in the rat. Life Sci. 34:197-201. 